\title{
Suppressor of cytokine signaling 1 expression during LPS-induced inflammation and bone loss in rats
}

João Antonio Chaves de SOUZA ${ }^{(a)}$ Andressa Vilas Boas NOGUEIRA ${ }^{(b)}$ Pedro Paulo Chaves de SOUZA(c) Guilherme José Pimentel Lopes de OLIVEIRA ${ }^{(b)}$

Marcell Costa de MEDEIROS(b) Gustavo Pompermaier GARLET ${ }^{(d)}$ Joni Augusto CIRELLI (b) Carlos ROSSA JUNIOR(b)

(a) Universidade Federal de Goiás - UFG, Faculty of Dentistry, Department of Periodontology, Goiânia, GO, Brazil.

(b) Universidade Estadual Paulista - UNESP, School of Dentistry at Araraquara, Department of Diagnosis and Surgery, Araraquara, SP, Brazil.

(c) Universidade Estadual Paulista - UNESP, School of Dentistry at Araraquara, Department of Physiology and Pathology, Araraquara, SP, Brazil.

(d) Universidade de São Paulo - USP, School of Dentistry at Bauru, Department of Biological Sciences, Bauru, SP, Brazil.

Declaration of Interests: The authors certify that they have no commercial or associative interest that represents a conflict of interest in connection with the manuscript.

\section{Corresponding Author:}

João Antonio Chaves de Souza

E-mail: joaoacsouza@gmail.com

htpps://doi.org/10.1590/1807-3107BOR-2017.vol31.0075

Submitted: Feb 14, 2017

Accepted for publication: June 26, 2017

Last revision: July 12, 2017
Abstract: This study aimed to characterize the dynamics of suppressor of cytokine signaling (SOCS1) expression in a rat model of lipopolysaccharideinduced periodontitis. Wistar rats in the experimental groups were injected three times/week with LPS from Escherichia coli on the palatal aspect of the first molars, and control animals were injected with vehicle (phosphate-buffered saline). Animals were sacrificed 7, 15, and 30 days after the first injection to analyze inflammation (stereometric analysis), bone loss (macroscopic analysis), gene expression (qRT-PCR), and protein expression/activation (Western blotting). The severity of inflammation and bone loss associated with LPS-induced periodontitis increased from day 7 to day 15, and it was sustained through day 30. Significant $(p<0.05)$ increases in SOCS1, RANKL, OPG, and IFN- $\gamma$ gene expression were observed in the experimental group versus the control group at day 15. SOCS1 protein expression and STAT1 and NF- $\mathrm{kB}$ activation were increased throughout the 30-day experimental period. Gingival tissues affected by experimental periodontitis express SOCS1, indicating that this protein may potentially downregulate signaling events involved in inflammatory reactions and bone loss and thus may play a relevant role in the development and progression of periodontal disease.

Keywords: Signal Transduction; Periodontitis; Lipopolysaccharides; Suppressor of Cytokine Signaling Proteins.

\section{Introduction}

Periodontal disease comprises a variety of conditions affecting the health of the periodontium, and it is highly prevalent in humans. ${ }^{1}$ Establishment of this disease depends both the presence of a biofilm and an unbalanced response by the host that may be affected by social and cultural behaviors, systemic conditions, and genetic and epigenetic traits. ${ }^{2}$ Among biofilm-derived factors, microbe-associated molecular patterns (MAMPs) deserve special attention due to their capacity to bind to pathogen recognition receptors expressed in host cells and induce inflammation and the expression of proinflammatory cytokines. ${ }^{3,4,5}$ The most studied MAMP, lipopolysaccharide (LPS), is expressed on the surface of gram-negative bacteria, and it acts by binding to TLR2 (Porphyromonas gingivalis LPS) and TLR4 (Escherichia coli LPS) on the surface of inflammatory and resident cells. ${ }^{6,7}$ 
The inflammatory microenvironment associated with the persistence of cytokines and other mediators results in the uncoupling of osteoblast and osteoclast function, together with increasing alveolar bone resorption and subsequent reduction of the supporting apparatus of the teeth. ${ }^{8}$ The increased expression of inflammatory cytokines during inflammation can affect the production and release of receptor activator of nuclear factor- $\mathrm{\kappa B}$ ligand (RANKL), a key regulator of osteoclast differentiation expressed by T-cells, stromal cells, osteoblasts, and osteocytes. ${ }^{9}$ RANKL binds to its receptor RANK expressed by hematopoietic precursors to stimulate osteoclastogenesis. ${ }^{10,11}$ The effects of RANKL are regulated by osteoprotegerin (OPG), which in turn inhibits osteoclast differentiation by preventing the binding of RANK and RANKL. ${ }^{12}$ Changes in the balance of these mediators define the severity and progression of periodontal disease..$^{13}$

Inflammatory mediators associated with periodontal disease may exert their biological effects by signaling through the Janus kinase (JAK)-signal transducer and activator of transcription (STAT) pathway. The binding of interferons, interleukins, and growth factors to their receptors activates JAK, which phosphorylates the cytoplasmic domain of the receptor to allow the recruitment and phosphorylation of STAT. Activated STATs dimerize and translocate to the nucleus, where they induce transcription and regulate target gene expression. ${ }^{14}$

Members of the suppressor of cytokine signaling (SOCS) family have been demonstrated to be inducible endogenous negative regulators of JAK-STAT signaling. These inducible proteins inhibit the dimerization of STATs downstream of the phosphorylation of receptor-associated JAK. ${ }^{15}$ SOCS proteins act through negative feedback of the JAK-STAT pathway, as their expression is also controlled by JAK-STAT activity. Among this group of proteins, SOCS1 and SOCS3 deserve special attention because of their confirmed relevance in limiting the progression of rheumatoid arthritis by inhibiting excessive bone resorption. ${ }^{16,17}$

SOCS1 and SOCS3 are upregulated in gingivitis and periodontitis, as well as in apical periodontitis in humans, suggesting that this endogenous mechanism of negative regulation of inflammation is activated under these conditions. ${ }^{18,19}$ Furthermore, a recent report identified SOCS3 as a critical negative regulator of alveolar bone loss in experimental periodontitis induced in SOCS-3-knockout mice. ${ }^{20}$ Supporting these findings, our group recently reported that SOCS1 and SOCS3 expression is correlated with the overall severity of inflammation and the expression of proinflammatory cytokines in a rat model of ligature-induced periodontitis. ${ }^{21}$ This model is marked by the presence of a plethora of bacterial virulence factors from endogenous bacterial species resulting in periodontal inflammation and bone loss. ${ }^{22}$ To analyze the participation of SOCS1 in periodontitis in a more controlled model, we investigated its expression in a model of periodontitis induced by LPS injection. This model enables the evaluation of host responses after stimulation of a specific TLR, facilitating control of the stimulus intensity, and it is independent of variables such as the colonization and survival of exogenous bacteria. ${ }^{22}$ These data will open new possibilities for investigating the mechanistic aspects of the participation of SOCS1 in periodontitis, especially regarding the responses to LPS.

\section{Methodology}

\section{Animal care and periodontitis induction}

The study protocol was approved by the Ethical Committee for Animal Experimentation (protocol number 23/2007) and conducted according to the ARRIVE guidelines. ${ }^{23}$ Thirty-six male adult Wistar (Rattus norvegicus albinus) rats weighing approximately $250 \mathrm{~g}$ were randomly divided into two experimental groups. In the experimental group ( $\mathrm{n}=9 /$ period), $30 \mu \mathrm{g}$ of Escherichia coli LPS (Strain 055: B5, Sigma-Aldrich) were injected into the palatal tissues between the first and second molars on both sides. The LPS injection was performed three times per week, whereas the control group $(n=9)$ received phosphate-buffered saline (vehicle) injections.

Three animals from the control group and nine animals from the experimental group were euthanatized at various time points $(7,15$, and 30 days after the first injection). The maxillary jaws of the animals were harvested, and one side was submitted to routine histological processing for 
use in stereometric and immunohistochemistry analyses. For the other side, the gingival tissue around the first molars was carefully dissected and immediately frozen in liquid nitrogen, followed by storage at $-80^{\circ} \mathrm{C}$ until the extraction of total mRNA (for qRT-PCR) and protein (for Western blotting). After the complete removal of soft tissues, these samples were maintained in $70 \%$ ethanol until the macroscopic analysis of bone loss.

\section{Macroscopic analysis of bone loss}

Bone loss at the palatal surface of the first molars was measured macroscopically ( $n=6$ per group). The samples were immersed for $5 \mathrm{~min}$ in a solution containing $0.7 \mathrm{~g} / \mathrm{L}$ methylene blue and washed with distilled water to remove excess stain.

Digital images of the palatal surfaces of the stained first and second molars were obtained at a standardized $90^{\circ}$ angle with the mid-palatal aspect of each molar using a stereomicroscope (Leica MZ6, $\times 20$ magnification). The total area of exposed cementum (CEJ-bone crest) was measured by an experienced and blinded examiner. Bone loss was calculated by subtracting the amount of exposed cementum in the experimental group from that in the control group at each time point. An increase in the area of exposed roots in comparison to that in control teeth indicates alveolar bone resorption.

\section{Stereometric analysis}

The samples collected for histology were fixed in $4 \%$ buffered formalin for $48 \mathrm{~h}$, decalcified in EDTA (0.5 M, pH 8.0) for 12 weeks, and embedded in paraffin ( $\mathrm{n}=6$ per group). Semi-serial sections ( $5 \mu \mathrm{m}$ thick) were obtained in the buccal-palatal direction and stained with hematoxylin/eosin. Photomicrographs of the sections were obtained using an optical microscope set at $\times 200$ magnification (Diastar-Cambridge Instruments) and a digital camera (Leica DFC 300 FX). A 32,400- $\mu \mathrm{m}^{2}$ grid consisting of $9 \times 4$ squares of $30 \mu \mathrm{m}$ was positioned in the bone crest area of the palatal surface using image-editing software (Adobe Photoshop CS5). Stereometric analysis was performed by a single blinded and experienced examiner (JACS) using a point-counting technique. The following structures observed at each intersection point of the grid were recorded: fibroblastic (elongated morphology) cells, vascular structures, inflammatory (rounded morphology) cells, and other structures. The presence of each structure was expressed as a percentage of the total area analyzed.

\section{Immunohistochemistry analysis}

Immunohistochemical analysis for TRAP was performed via the avidin-biotin peroxidase method using an LSAB kit according to manufacturer's instructions (Dako, Glostrup, Denmark). Six samples per group were incubated overnight with TRAP antibody diluted at 1:300 (sc-30833, Santa Cruz Biotechnology). Slides were stained with 3,3'-diaminobenzidine and counterstained with Carazzi's hematoxylin. To perform osteoclast quantification using TRAP staining, histological slides of the furcation area were evaluated under $\times 400$ magnification. TRAP+ cells containing two or more nuclei present in the vicinity of the bone surface were considered osteoclasts. The number of osteoclasts in a furcation area in the total area of $32,400 \mu \mathrm{m}^{2}$ was counted by a trained examiner blind to the experimental groups.

\section{qRT-PCR}

Total RNA from gingival tissues was extracted $(n=6$ per group) using a specific kit (RNAqueous-4PCR kit, Thermo Fisher). Four hundred nanograms of total RNA were converted into cDNA using reverse transcriptase in a total volume of $20 \mu \mathrm{L}$ (High Capacity cDNA kit, Applied Biosystems).

qRT-PCR was performed using TaqMan qPCR master mix (Applied Biosystems), cDNA, deionized water, and species-specific pre-designed and optimized pairs of primers and probes (TaqMan gene expression assays) (Table). The relative gene expression level was determined using the cycle threshold method and normalized to the housekeeping gene Gapdh. The mean Ct values of duplicate measurements was used to calculate the expression of the target genes using the normalization formula $2^{-} \Delta \Delta^{\mathrm{Ct}}$. Results were expressed as fold change over the expression levels of the normalized target gene determined in cDNA samples prepared from healthy gingival tissues (control group). 
Table. Inventoried TaqMan primers and probe (TaqMan Gene Expression Assays, Applied Biosystems).

\begin{tabular}{lccc}
\hline Target gene & Assay ID & Accession \# & Amplicon (bp) \\
\hline GAPDH & Rn99999916_sl & NM_017008.3 & 87 \\
RANKL & Rn00589289_ml & NM_057149.1 & 69 \\
OPG & Rn00563499_ml & NM_012870.2 & 75 \\
INF- $\gamma$ & Rn00594078_ml & NM_138880.2 & 91 \\
SOCS1 & Rn00595838_sl & NM_145879.1 & 76 \\
\hline
\end{tabular}

\section{Western blot}

SOCS1 expression and STAT1 and NF-kB activation were assessed using total protein samples extracted from gingival tissues ( $\mathrm{n}=6$ per group/period). Proteins were extracted using a detergent-based extraction buffer (T-PER, Pierce) in the presence of protease and phosphatase inhibitors, and the total protein concentration was measured using a Bradford assay (Bio-Rad Laboratories, City, Country). Forty micrograms of total protein in each sample were resolved by SDS-acrylamide gel electrophoresis and electrotransferred to nitrocellulose membranes. For detection, after blocking in 2\% BSA for $2 \mathrm{~h}$ (room temperature), the membranes were incubated overnight at $4^{\circ} \mathrm{C}$ with primary antibodies (Santa Cruz Biotechnology, City, Country) diluted at 1:100 (p-STAT1), 1:200 (STAT1, SOCS1, p65 NF-кB, p50 NF-кB), or 1:500 (GAPDH). Afterward, the membranes were incubated with species-specific secondary antibodies conjugated with HRP (1:2000 dilution). Detection of target proteins was performed on radiographic film using a chemiluminescence system (Pierce ECL Substrate). Digitalized images of the radiographic films of six independent samples for each period were obtained using a documentation system (ImageQuant 100, GE Lifesciences, City, Country), and densitometric measurements were obtained using Image 1 1.5i software (NIH, Bethesda, USA). The density of the target proteins was normalized to the density of GAPDH and expressed in arbitrary densitometric units.

\section{Data analysis}

The qRT-PCR and stereometric data were statistical analyzed using one-way ANOVA followed by Tukey's post hoc test. Statistical analysis of densitometric data for Western blotting was performed using an unpaired $t$-test with Welch's correction for unequal variances. The associations between mRNA SOCS1 and cytokine gene expression were assessed using Pearson's correlation coefficients. All tests used a significance level of $5 \%$.

\section{Results}

\section{Stereometry, bone loss, and TRAP}

The results of the histologic analysis are presented in Figure 1. There were increases in the numbers of inflammatory cells and vascular structures and decreases in the number of fibroblasts in the experimental group. The severity of inflammation was higher in the 30-day experimental period than in the control group. Corroborating these findings, alveolar bone loss and increases in the number of osteoclasts occurred as early as 7 days, followed by significant increases after 15 and 30 days (Figure 2).

\section{PCR and Western blotting}

After 15 and 30 days, the mRNA expression of RANKL, OPG, and IFN- $\gamma$ was significantly increased compared with that in the control group ( $p<0.05)$, with expression peaking at 15 days (Figure $3 \mathrm{~A}-\mathrm{C}$ ). The gene expression of SOCS1 paralleled that of inflammatory cytokines and also peaked at 15 days (Figure3 D). There were significant positive correlations $(p<0.05)$ between SOCS1 and RANKL mRNA levels (Figure 3 E) and between SOCS1 and IFN- $\gamma$ mRNA (Figure $3 \mathrm{~F}$ ). However, it is important to know that these correlations do not establish cause-effect links; conversely, they demonstrate that different molecules may be regulated simultaneously.

In addition, Western blot analysis revealed increased protein expression of SOCS1 in animals with LPS-induced periodontitis in all periods (Figure 4). Moreover, activation of STAT1 and NF- $\mathrm{B}$ followed the expression of SOCS1 protein, beginning at 7 days and peaking at 15 days (Figure 4).

\section{Discussion}

The injection of LPS into the gingival tissue of rats is a frequently and well-described model to study periodontitis that enables the evaluation 
A

CONTROL - 7 days

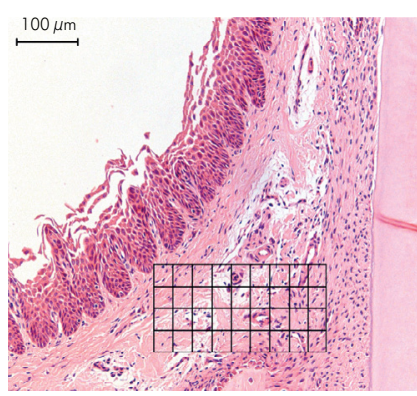

E

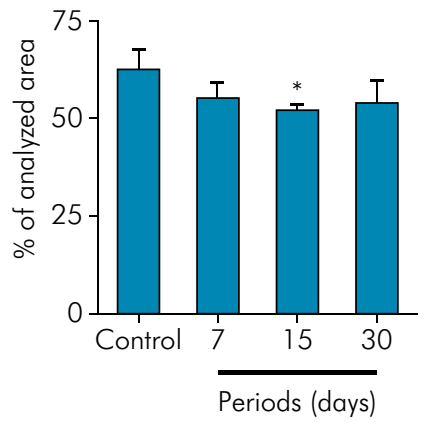

B $\quad 7$ days

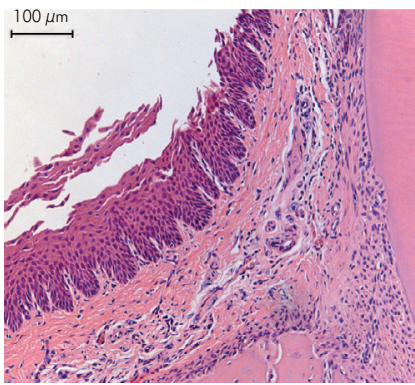

$\mathbf{F}$

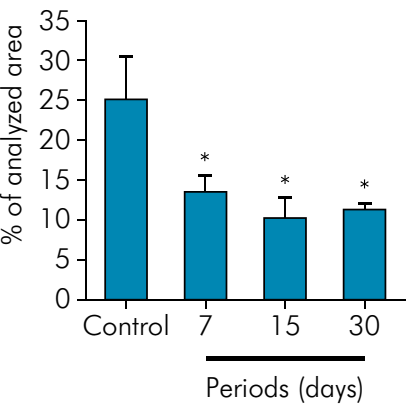

C $\quad 15$ days

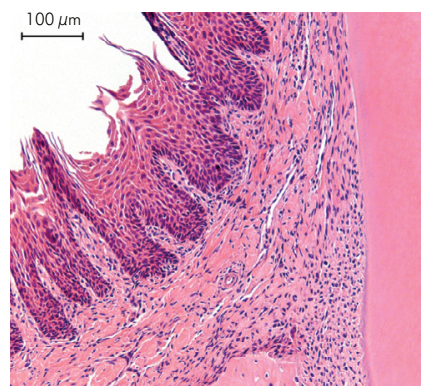

G

Inflammatory cells

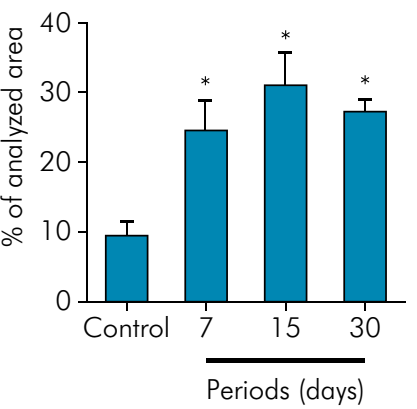

D $\quad 30$ days

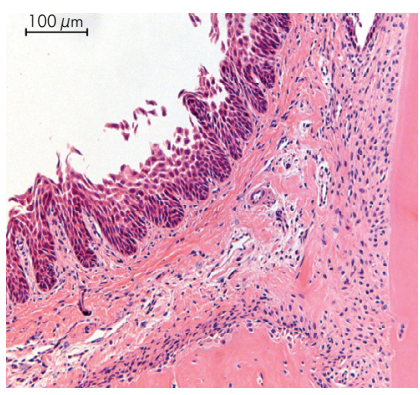

H Vascular structures

* Significant difference compared with the control $(p<0.05)$.

Figure 1. Photomicrographs of sections of the bone crest area in the control $(A)$ and experimental groups $(B, C, D)$ were analyzed. A single representative image of the control group is shown, as no important changes were noted in this group during the experimental period (data not shown). The presence of inflammatory cells $(G)$, vascular structures $(H)$, fibroblasts $(F)$, and other structures (E) in a $32,400-\mu \mathrm{m}^{2}$ area was analyzed using a point-counting technique, revealing increases in inflammatory cell density and vascular structures and decreases in the number of fibroblasts and other structures in diseased samples in all experimental periods. Inflammation was significantly more severe in the experimental groups than in the control group.

of host responses after the specific stimulation of TLRs using a controlled intensity of stimulus, that is independent of variables such as the colonization and survival of exogenous bacteria. LPS from Escherichia coli (TLR4 agonist) injections in this model induce the production of inflammatory cytokines through the activation of several signaling pathways including JAK-STAT, MAPK, and NF- $\kappa$ B, leading to inflammatory responses and bone loss. ${ }^{24}$ SOCS1 has been identified as a negative regulator of TLR4 signaling; however, this mechanism is not fully understood. ${ }^{25,26}$ The main role of SOCS1 is to downregulate the intracellular signaling of inflammatory mediators via JAK-STAT.

Previously, our group analyzed SOCS1 expression in rats with ligature-induced periodontitis. At the protein level, the expression of SOCS1 accompanied the progression and severity of periodontitis in that model, increasing through 7 days before gradually decreasing after 15 and 30 days. ${ }^{21}$ In contrast with these data, we demonstrated that SOCS1 upregulation is sustained in the gingiva of rats after 15 and 30 days of LPS exposure. These conflicting data can be explained by differences between the experimental models. In the ligature model, the progression of periodontitis is blunted due to apical migration of the junctional epithelium and initial bone resorption, which distances the supporting apparatus from the pathogenic stimulus, thus allowing the initiation of repair. In the LPS model, the disease is sustained due to the sustained presence of the stimulus via continuous injections during the 30-day experimental period. Thus, SOCS1 expression is dependent on the presence of the stimulus, as it decreases if the stimulus is removed. 
A

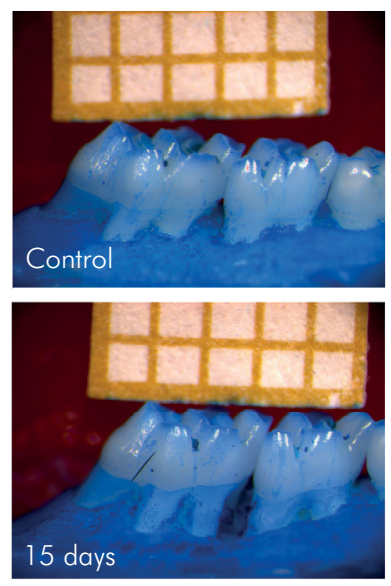

B
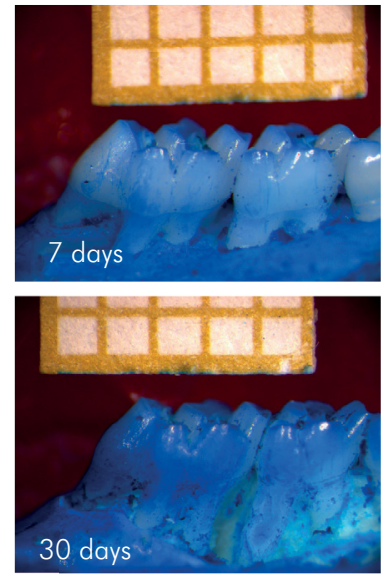
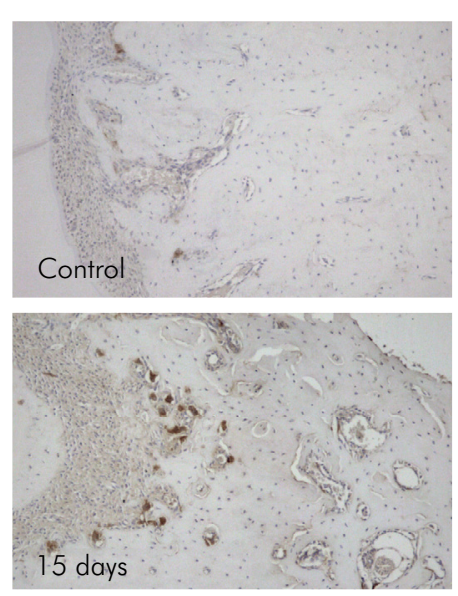
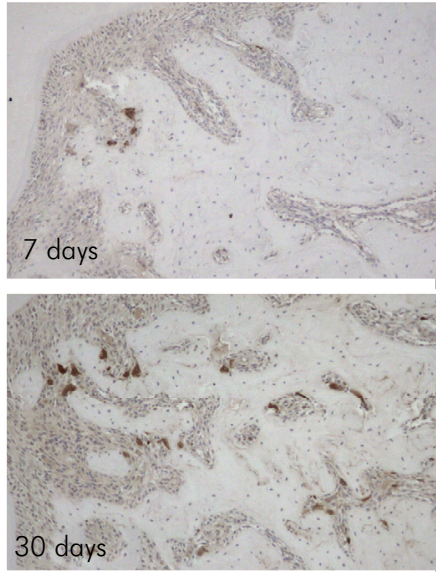
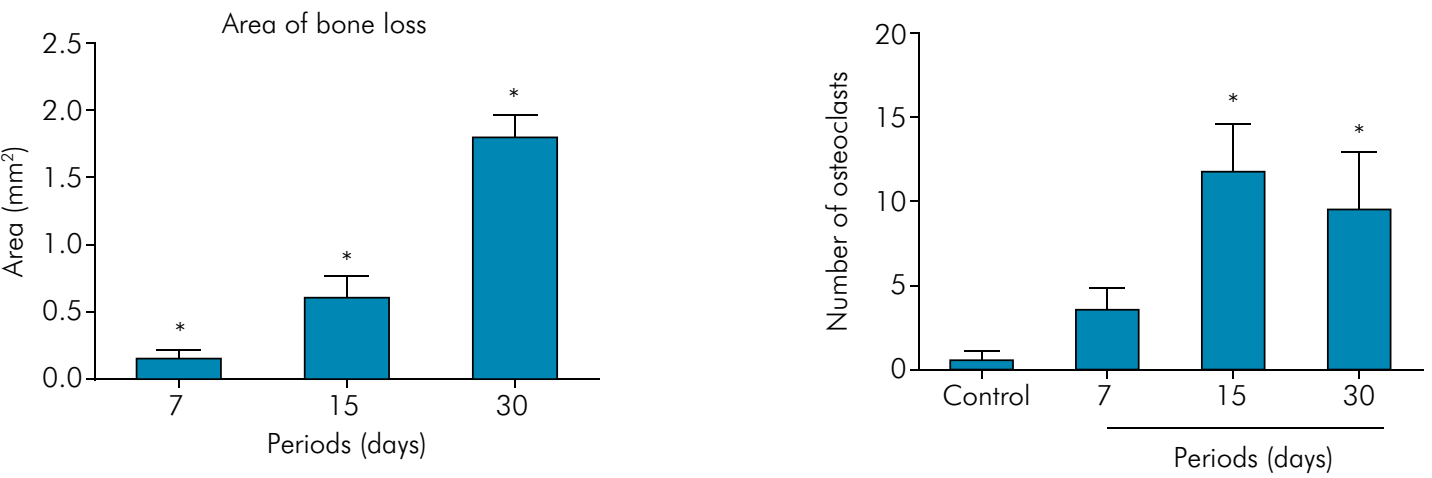

* Significant difference $(p<0.05)$ compared with the control.

Figure 2. (A) The area of bone loss at the palatal surface of the first molar was measured macroscopically. Samples stained with methylene blue are shown for each group. The graph with average \pm standard deviation demonstrated that increased bone loss occurred as early as 7 days, followed by a significant increase after 15 and 30 days. (B) Representative images $(\times 100$ magnification) of immunohistochemical staining for TRAP showing osteoclasts in tissue sections from the control and experimental groups. The graph revealed increases in osteoclast counts throughout the 30-day experimental period, peaking at 15 days, in comparison to the control group findings.

SOCS proteins are attenuators of cytokine-induced inflammatory processes mediated by the JAK/STAT pathway, and their increased expression can control signal transduction. On the contrary, SOCS expression is upregulated by cytokines, triggering the activation of JAK-STAT signaling as part of a negative feedback mechanism that inhibits excessive inflammatory responses. ${ }^{27}$ Therefore, in accordance with our results, SOCS1 expression is minimal in healthy tissues and high in inflamed tissues. ${ }^{18}$

SOCS1 is a negative regulator of immune mechanisms in inflammatory diseases, and its deficiency results in increased tissue destruction. ${ }^{17}$ Thus, SOCS1 expression in periodontal disease may be of importance for limiting tissue degradation. Our in vivo results corroborate clinical findings that SOCS1 is highly expressed in patients with periodontitis, who also exhibit increased levels of inflammatory cytokines, in comparison to its expression in healthy controls..$^{18}$ In our model, we also observed that SOCS1 expression is strongly associated with the inflammatory condition, as evidenced by our stereometric analysis. Therefore, we suggest that SOCS1 production is induced by cytokines and other biological mediators released by inflammatory and resident cells during periodontal disease induction. This could explain the induction of SOCS1 expression and concomitant gene expression of proinflammatory cytokines and RANKL. In addition to 
the expression of cytokines described in our previous study such as IL-6, TNF- $\alpha$, and IL-1 $\beta^{28}$, we analyzed IFN-y, RANKL, and OPG. As expected, the mRNA expression of these inflammatory cytokines paralleled
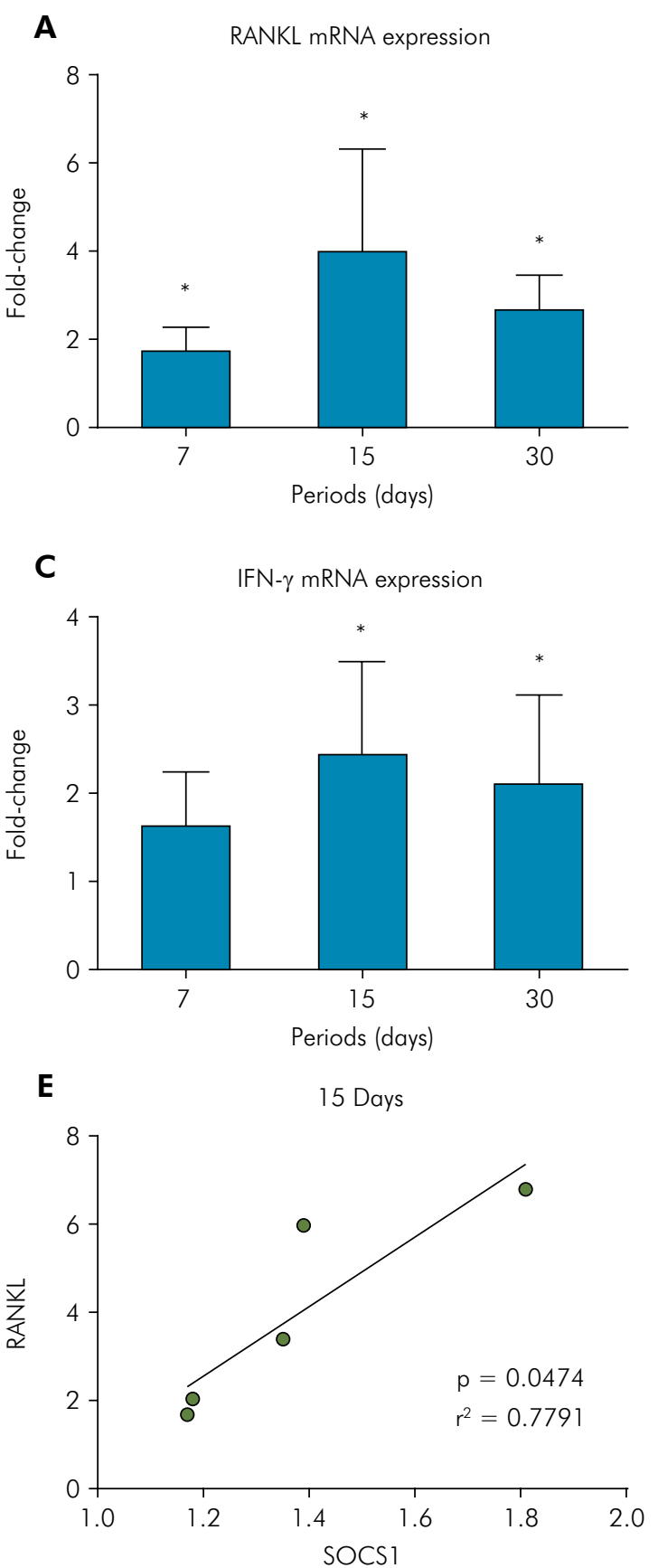

the expression of SOCS1. This is in agreement with previous investigations illustrating that SOCS1 is induced by various cytokines, including IFN- $\gamma$ and RANKL. ${ }^{25,29}$
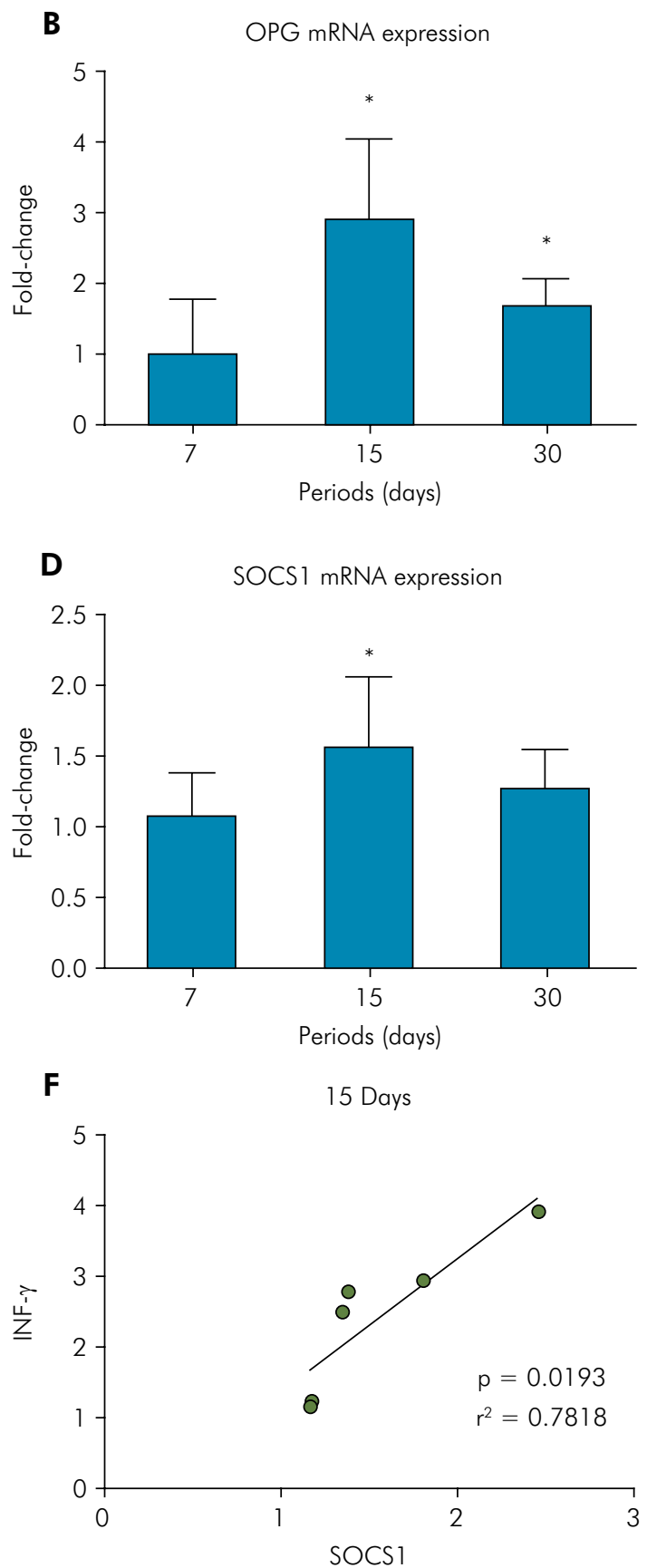

* Significant difference $(p<0.05)$ compared with the control.

Figure 3. Expression of SOCSI (E), RANKL (A), OPG (B), and INF- $\gamma(C)$ mRNA during the course of LPS-induced periodontitis was increased after 7,15 , and 30 days of LPS exposure, peaking at 15 days. The bars represent the mean fold change in comparison to the untreated control, and the vertical lines denote the standard deviation. There were significant positive correlations $(p<0.05)$ between SOCSI and RANKL mRNA expression (E) and between SOCSI and IFN- $\gamma$ mRNA expression (F). 
A
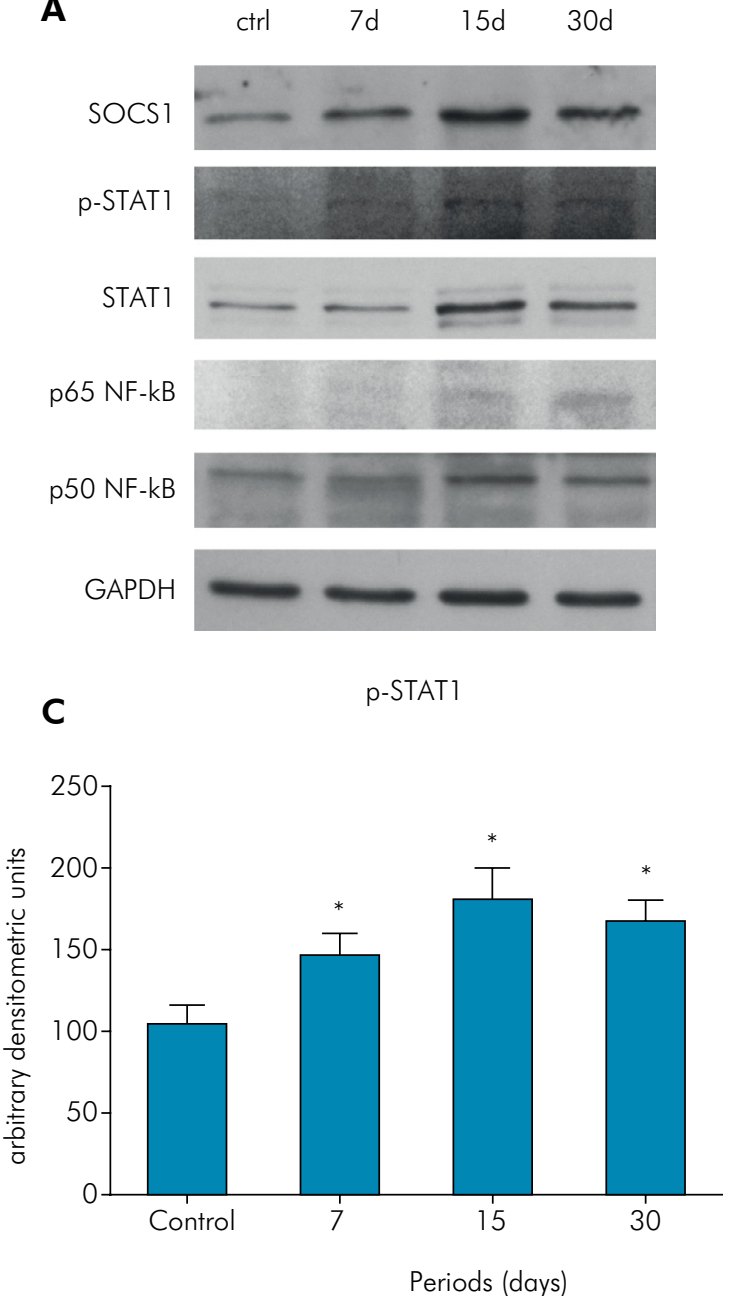

$\mathbf{E}$

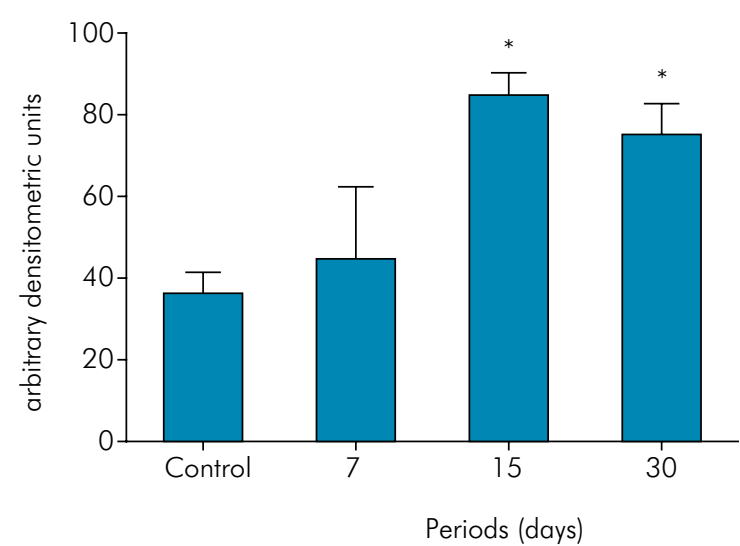

B

SOCS1

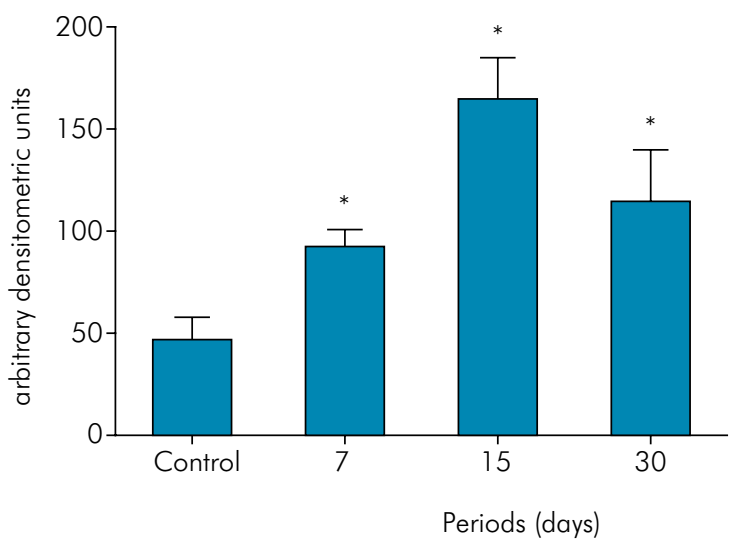

D STAT1

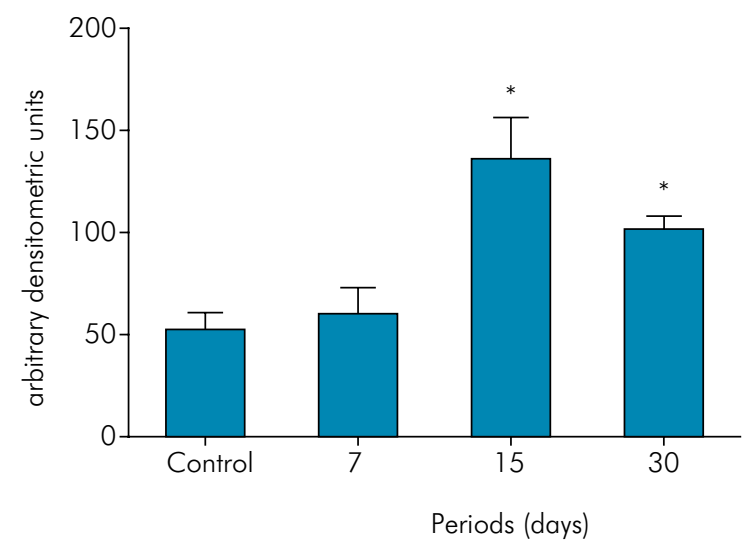

p50 NF-kB

$\mathbf{F}$

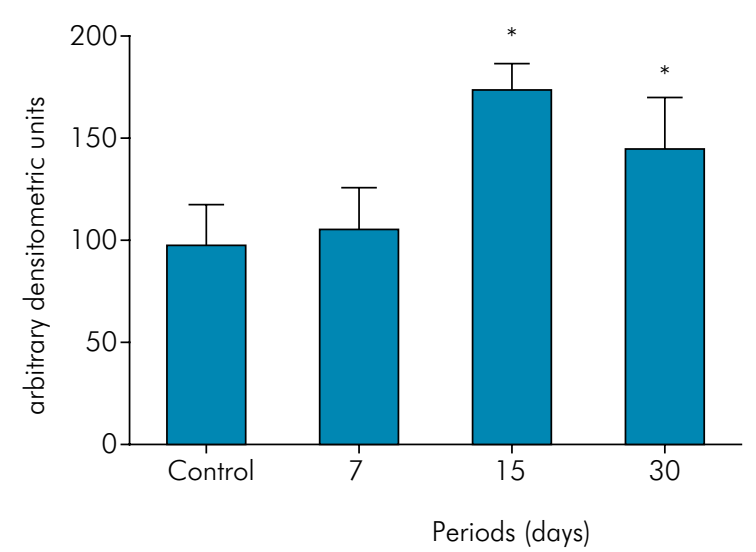

* Significant difference $(\mathrm{p}<0.05)$ compared with the control.

Figure 4. (A) Western blot analysis revealed that SOCS1 expression and STAT1 and NF-אB activation were increased after 7 days in the experimental group, peaking at 15 days. Densitometric analysis of the Western blot results indicated sustained activation of SOCSI (B), STATI (C, D), and NF-KB (E, F) at LPS injection sites beginning at day 7 and peaking at day 15. The bars represent the mean normalized arbitrary densitometric units of six independent samples for each period, and vertical lines show the standard deviation. 
SOCS proteins are believed to regulate cytokine signaling and consequently influence inflammation-induced bone loss. Therefore, SOCS can counteract the effects of cytokines that promote inflammation-induced bone loss, thus preventing excessive inflammatory damage to host tissues. ${ }^{30}$ Data from the literature indicate thatSOCS1 plays critical roles in osteoclastogenesis in pathological situations both in vitro and in vivo. ${ }^{29}$ Interestingly, our results revealed that SOCS1 expression mirrors the degree/severity of inflammation and bone loss/number of osteoclasts during periodontal disease progression.

SOCS1 protein acts as an endogenous negative regulator of STAT1, primarily affecting the JAK-STAT pathway. ${ }^{14,15}$ In our results, we observed that SOCS1 protein expression mirrors STAT1 and NF-KB expression in its active forms. This result is consistent with literature demonstrating that NF- $\kappa B$ is also regulated by SOCS1. ${ }^{31}$ SOCS1 regulates TLR-induced NF- $\mathrm{KB}$ activation via a direct interaction and degradation of the p65 subunit of NF- $\mathrm{KB}$ upon stimulation with LPS. ${ }^{32}$ Furthermore, through a negative feedback mechanism, increases of STAT protein activity lead to increased SOCS expression in an attempt to reduce activation of the JAK/STAT pathway, diminishing the consequences of the inflammatory response. These data suggest that SOCS proteins may be important factors in the regulation of genes associated with the degradation and resorption of bone and connective tissue in periodontal disease. This knowledge will be further explored in gain- and loss-of-function experiments, which in turn may lead to a novel immunomodulatory therapeutic approach using synthetic peptides mimicking SOCS1-binding domains.

\section{Conclusion}

The results obtained in this study demonstrated that gingival tissues affected by LPS-induced experimental periodontitis express SOCS1 and that this expression parallels the severity of inflammation. This indicates that SOCS1 may potentially downregulate signaling involved in inflammatory reactions and bone loss, and thus, it may play a relevant role in the development and progression of periodontal disease. Additional experiments including deletion, inhibition, and upregulation of SOCS1 are required to determine the function of SOCS proteins in the modulation of host responses associated with periodontal disease and verify possible novel targets of these proteins. Further understanding of the mechanisms by which SOCS1 participates in periodontal tissue destruction in patients affected for periodontal disease will be needed to determine its therapeutic potential.

\section{Acknowledgments}

The authors want to express their gratitude to research technician Ana Claudia Gregolin da Costa Miranda (Department of Diagnosis and Surgery, UNESP at Araraquara) for technical assistance.

\section{References}

1. Eke PI, Dye BA, Wei L, Thornton-Evans GO, Genco RJ, Cdc Periodontal Disease Surveillance workgroup: James Beck GDRP. Prevalence of periodontitis in adults in the United States: 2009 and 2010. J Dent Res. 2012;91(10):914-20. https://doi.org/10.1177/0022034512457373

2. Bartold PM, Van Dyke TE. Periodontitis: a host-mediated disruption of microbial homeostasis. Unlearning learned concepts. Periodontol 2000. 2013;62(1):203-17. https://doi.org/10.1111/j.1600-0757.2012.00450.x

3. Kawai T, Akira S. The role of pattern-recognition receptors in innate immunity: update on Toll-like receptors. Nat Immunol. 2010;11(5):373-84. https://doi.org/10.1038/ni.1863
4. Beutler B. LPS in microbial pathogenesis: promise and fulfilment. J Endotoxin Res. 2002;8(5):329-35. https://doi.org/10.1179/096805102125000650

5. Beutler B. Science review: key inflammatory and stress pathways in critical illness: the central role of the Toll-like receptors. Crit care. 2003;7(1):39-46. https://doi.org/10.1186/cc1828

6. Kassem A, Henning P, Lundberg P, Souza PP, Lindholm C, Lerner UH. Porphyromonas gingivalis Stimulates Bone Resorption by Enhancing RANKL (Receptor Activator of NF-kB Ligand) through Activation of Toll-like Receptor 2 in Osteoblasts. J Biol Chem. 2015;290(33):20147-58. https://doi.org/10.1074/ibc.M115.655787 
7. Nakamura H, Fukusaki Y, Yoshimura A, Shiraishi C, Kishimoto M, Kaneko T, et al. Lack of Toll-like receptor 4 decreases lipopolysaccharide-induced bone resorption in $\mathrm{C} 3 \mathrm{H} / \mathrm{HeJ}$ mice in vivo. Oral Microbiol Immunol. 2008;23(3):190-5. https://doi.org/10.1111/j.1399-302X.2007.00410.x

8. Souza PP, Lerner UH. The role of cytokines in inflammatory bone loss. Immunol Invest. 2013;42(7):555-622. https://doi.org/10.3109/08820139.2013.822766

9. Boyce BF, Xing L. Functions of RANKL/RANK/OPG in bone modeling and remodeling. Arch Biochem Biophys. 2008;473(2):139-46. https://doi.org/10.1016/i.abb.2008.03.018

10. Yamano E, Miyauchi M, Furusyo $H$, Kawazoe A, Ishikado A, Makino $T$, et al. Inhibitory effects of orally administrated liposomal bovine lactoferrin on the LPS-induced osteoclastogenesis. Lab Invest. 2010;90(8):1236-46. https://doi.org/10.1038/labinvest.2010.80

11. Cochran DL. Inflammation and bone loss in periodontal disease. J Periodontol. 2008;79(8 Suppl):1569-76. https://doi.org/10.1902/jop.2008.080233

12. Katagiri T, Takahashi N. Regulatory mechanisms of osteoblast and osteoclast differentiation. Oral Dis. 2002;8(3):147-59. https://doi.org/10.1034/j.1601-0825.2002.01829.x

13. Graves DT, Cochran D. The contribution of interleukin-1 and tumor necrosis factor to periodontal tissue destruction. Journal of periodontology. 2003;74(3):391-401. https://doi.org/10.1902/jop.2003.74.3.391

14. Starr R, Hilton DJ. Negative regulation of the JAK/STAT pathway. BioEssays. 1999;21(1):47-52. https:// doi.org/10.1002/(SICI)1521-1878(199901)21:1<47::AIDBIES6>3.0.CO;2-N

15. Yoshimura A, Naka T, Kubo M. SOCS proteins, cytokine signalling and immune regulation. Nat Rev Immunol. 2007;7(6):454-65. https://doi.org/10.1038/nri2093

16. Veenbergen S, Bennink MB, Affandi AJ, Bessis N, Biton J, Arntz OJ et al. A pivotal role for antigen-presenting cells overexpressing SOCS3 in controlling invariant NKT cell responses during collagen-induced arthritis. Ann Rheum Dis. 2011;70(12):2167-75. https://doi.org/10.1136/ard.2011.154815

17. Egan PJ, Lawlor KE, Alexander WS, Wicks IP. Suppressor of cytokine signaling- 1 regulates acute inflammatory arthritis and T cell activation. J Clin Invest. 2003;111(6):915-24. https://doi.org/10.1172/JCI16156

18. Garlet GP, Cardoso CR, Campanelli AP, Martins W, Jr., Silva JS. Expression of suppressors of cytokine signaling in diseased periodontal tissues: a stop signal for disease progression? J Periodontal Res. 2006;41(6):580-4. https://doi.org/10.1111/j.1600-0765.2006.00908.x

19. Menezes R, Garlet TP, Trombone AP, Repeke CE, Letra A, Granjeiro JM, et al. The potential role of suppressors of cytokine signaling in the attenuation of inflammatory reaction and alveolar bone loss associated with apical periodontitis. J Endod. 2008;34(12):1480-4. https://doi.org/10.1016/i.joen.2008.09.003
20. Papathanasiou E, Kantarci A, Konstantinidis A, Gao H, Van Dyke TE. SOCS-3 Regulates alveolar bone loss in experimental periodontitis. J Dent Res. 2016;95(9):1018-25. https://doi.org/10.1177/0022034516645332

21. Souza JA, Nogueira AV, Souza PP, Cirelli JA, Garlet GP, Rossa C Jr. Expression of suppressor of cytokine signaling 1 and 3 in ligature-induced periodontitis in rats. Arch Oral Biol. 2011;56(10):1120-8. https://doi.org/10.1016/i.archoralbio.2011.03.022

22. Graves DT, Kang J, Andriankaja O, Wada K, Rossa C, Jr. Animal models to study host-bacteria interactions involved in periodontitis. Front Oral Biol. 2012;15:117-32. https://doi.org/10.1159/000329675

23. Kilkenny C, Browne WJ, Cuthill IC, Emerson M, Altman DG. Improving bioscience research reporting: the ARRIVE guidelines for reporting animal research. Osteoarthritis Cartilage. 2012;20(4):256-60. https://doi.org/10.1016/i.joca.2012.02.010

24. Aquino SG, Leite FRM, Stach-Machado DR, Silva JAF, Spolidorio LC, Rossa Junior C. Signaling pathways associated with the expression of inflammatory mediators activated during the course of two models of experimental periodontitis. Life Sci. 2009;84(21-22):745-54. https://doi.org/10.1016/i.lfs.2009.03.001

25. Naka T, Fujimoto $M$, Tsutsui $H$, Yoshimura A. Negative regulation of cytokine and TLR signalings by SOCS and others. Adv Immunol. 2005;87:61-122. https://doi.org/10.1016/S0065-2776(05)87003-8

26. Fujimoto $M$, Naka T. SOCS1, a negative regulator of cytokine signals and TLR responses, in human liver diseases. Gastroenterology research and practice. 2010;2010:ID470468. https://doi.org/10.1155/2010/470468

27. Alexander WS, Hilton DJ. The role of suppressors of cytokine signaling (SOCS) proteins in regulation of the immune response. Annu Rev Immunol. 2004;22(1):503-29. https://doi.org/10.1146/annurev.immunol.22.091003.090312

28. Souza JAC, Nogueira AV, Souza PPC, Kim YJ, Lobo CS, Oliveira GJPL et al. SOCS3 expression correlates with severity of inflammation, expression of proinflammatory cytokines, and activation of STAT3 and p38 MAPK in LPS-induced inflammation in vivo. Mediators Inflamm. 2013;2013:ID650812. https://doi.org/10.1155/2013/650812

29. Ohishi M, Matsumura Y, Aki D, Mashima R, Taniguchi K, Kobayashi T et al. Suppressors of cytokine signaling- 1 and -3 regulate osteoclastogenesis in the presence of inflammatory cytokines. J Immunol. 2005;174(5):3024-31. https://doi.org/10.4049/jimmunol.174.5.3024

30. Yoshimura A, Nishinakamura H, Matsumura Y, Hanada T. Negative regulation of cytokine signaling and immune responses by SOCS proteins. Arthritis Res Ther. 2005;7(3):100-10. https://doi.org/10.1186/ar1741 
31. Andrés MC, Imagawa K, Hashimoto K, Gonzalez A, Goldring MB, Roach $\mathrm{HI}$ et al. Suppressors of cytokine signalling (SOCS) are reduced in osteoarthritis.

Biochem Biophys Res Commun. 2011;407(1):54-9.

https://doi.org/10.1016/j.bbrc.2011.02.101
32. Ryo A, Suizu F, Yoshida Y, Perrem K, Liou YC, Wulf G, et al. Regulation of NF-kappaB signaling by Pinl-dependent prolyl isomerization and ubiquitin-mediated proteolysis of p65/RelA. Mol Cell. 2003;12(6):1413-26. https://doi.org/10.1016/S1097-2765(03)00490-8 